\title{
Physicians and Implicit Bias
}

\author{
Norman M. Jensen \\ Department of Medicine and Chair, American Academy on Communication in Healthcare, University of Wisconsin, Madison, USA.
}

J Gen Intern Med 29(5):707

DOI: $10.1007 / \mathrm{s} 11606-014-2793-1$

(c) Society of General Internal Medicine 2014

To the Editors:- The "Perspective" article by Chapman et al. ${ }^{1}$ helps me see how stereotype and implicit bias influences my clinical caring behavior, and unintentionally enables my contribution to health disparities. Their recommendation for "individuating and perspective taking" helps me structure a model for improvement-I've learned from 40 years of practice that the better I know the life story of my patient, the more invested I become and the stronger are my helping attitudes (empathy, congruence, and unconditional positive regard). ${ }^{2}$ I understand this as an example of "individuating" that enables "perspective taking" (empathy), thus facilitating an enhanced sense of identity (common humanity) with my patient. $^{3}$ A recently published randomized controlled trial (RCT) of cognitive restructuring resulted in a worthwhile small (effect size $\sim 0.4)$ 8-week-durable reduction in implicit bias and a larger $(\sim 0.6)$ increased concern about personal prejudice that grew over time. ${ }^{4}$ Such results can inform evidence-based professional education.

And, because explicit and implicit biases about the "other" likely carry strong affective valences, a robust debiasing of social thought and action will likely need enhanced emotional and cognitive intelligence. It may need enhanced self-awareness of motivating affects, such as a fear of the other. Full perspective taking may need active hearing and understanding the other's suffering from prejudice. It may need repetitive reflection in and on experience. All this is imaginable within current formats of clinical education.

Conflict of Interest: None.

Corresponding Author: Norman M. Jensen, Department of Medicine and Chair, American Academy on Communication in Healthcare University of Wisconsin, Madison, USA (e-mail: nmj@medicine.wisc.edu).

\section{REFERENCES}

1. Chapman EN, Kaatz A, Carnes M. Physicians and implicit bias: how doctors may unwittingly perpetuate health care disparities. JGIM. 2013;128:1504.

2. Rogers $\mathbf{C}$. The necessary and sufficient conditions of therapeutic personality change. J Consult Psychol. 1957;21:95-103.

3. Galinsky AD, Moskowitz GB. Perspective-taking: decreasing stereotype expression, stereotype accessibilit6y, and in-group favoritism. J Pers Soc Psychol. 2000;78:708-724.

4. Devine PG, et al. Long-term reduction in implicit race bias: A prejudice habit-breaking intervention. J Exp Soc Psychol. 2012;48:1267-1278. 